\title{
Remote Electrical Neuromodulation (REN) for the Acute Treatment of Menstrual Migraine: a Retrospective Survey Study of Effectiveness and Tolerability
}

\author{
Hida Nierenburg · Liron Rabany · Tamar Lin • Roni Sharon • \\ Dagan Harris · Alon Ironi · Paul Wright · Linus Chuang
}

Received: March 29, 2021 / Accepted: May 26, 2021 / Published online: June 17, 2021

(C) The Author(s) 2021

\section{ABSTRACT}

Introduction: Migraine is one of the most prevalent neurological disorders worldwide, and estimations are that $60 \%$ of women who suffer from migraines experience attacks that are associated with menstruation. Menstrual migraines are typically more debilitating and less responsive to pharmacological treatment. Remote electrical neuromodulation (REN) is a non-pharmacological abortive treatment of migraine headache. The current study evaluated the self-reported effectiveness and tolerability of REN for the acute treatment of menstrual

Supplementary Information The online version contains supplementary material available at https:// doi.org/10.1007/s40122-021-00276-7.

H. Nierenburg $(\varangle) \cdot$ P. Wright

Division of Neurology, Nuvance Health, Poughkeepsie, NY, USA

e-mail: hida.nierenburg@nuvancehealth.org

L. Rabany · T. Lin · D. Harris · A. Ironi

Theranica Bio-Electronics, Netanya, Israel

R. Sharon

Headache and Facial Pain, Sheba Medical Center, Ramat Gan, Israel

L. Chuang

Department of Obstetrics, Gynecology and Reproductive Biology, Danbury Hospital, Nuvance Health, 24 Hospital Avenue, Danbury, CT 06810, USA migraine, via a retrospective structured survey that was sent to adult female REN users.

Methods: Women aged 18-55 years who experience menstrually related or pure menstrual migraine and have completed at least four REN treatments, participated in this retrospective, observational survey study. Participants completed a short online survey assessing effectiveness, satisfaction, and safety outcomes.

Results: Ninety-one participants qualified for the analysis, out of which $74.7 \% \quad(68 / 91)$ reported that the treatment was at least moderately effective (moderately effective 37.4\%, very effective $26.4 \%$, extremely effective $11.0 \%$ ). Additionally, 45.1\% (41/91) reported satisfaction from REN (slightly satisfied 33\%, extremely satisfied $12.1 \%$ ), while $34.1 \%$ were neutral and 20.9\% (19/91) were not satisfied. Lastly, $100 \%$ of the participants reported that the treatment is at least moderately tolerable (moderately tolerable $8.8 \%$, very tolerable $20.9 \%$, extremely tolerable $70.3 \%$ ), and $13.2 \%$ (12/91) of respondents reported mild short-term side effects.

Conclusions: Nearly 75\% reported that the treatment was at least moderately effective, $45 \%$ reported satisfaction, and $100 \%$ of the participants reported that the treatment is at least moderately tolerable. Thirteen percent reported mild short-term side effects. REN was thus reported as effective for menstrual migraine by most participants and was very well tolerated. Therefore, REN may provide a safe, non- 
pharmacological alternative for the acute treatment of menstrual migraine.

\section{ClinicalTrial.GOV}

Number: NCT04600388.

Keywords: Migraine; Headache; Menstrual pain; Menstruation; Treatment; Analgesia; Nerivio; REN; Remote electric neuromodulation; Women's health

\section{Key Summary Points}

Why carry out this study?

Migraines associated with menstruation are typically more debilitating than nonmenstrual migraines, of longer duration, and less responsive to pharmacological treatments.

Remote electrical neuromodulation (REN) is a non-pharmacological abortive treatment for migraine headache.

The current study evaluated the selfreported effectiveness and tolerability of REN for the abortive treatment of menstrual migraine.

\section{What was learned from the study?}

REN was reported effective for menstrual migraine by most participants, and was very well tolerated.

The study suggests that REN may provide a safe, non-pharmacological alternative for the acute treatment of menstrual migraine.

\section{DIGITAL FEATURES}

This article is published with digital features, including a summary slide, to facilitate understanding of the article. To view digital features for this article go to https://doi.org/10.6084/ m9.figshare.14673303.

\section{INTRODUCTION}

Migraine is the world's second cause of years lived with disability, and the first cause among young women [1]. It is characterized by recurrent headache attacks that are often accompanied by nausea, vomiting, photophobia, and phonophobia [2]. About $60 \%$ of women who suffer from migraines experience attacks that are associated with menstruation [3]. Menstrual migraine is often more painful, more disabling, of longer duration, and less responsive to treatment [4].

Menstrual migraine is mainly managed by the same acute treatments as those used for non-menstrual migraine, including triptans and non-steroidal anti-inflammatory drugs (NSAIDs) [5]. However, these treatments have been shown to be less effective for menstrual migraines [6], thus possibly resulting in more frequent use and higher doses, which in turn are associated with medication overuse headache and migraine chronification [7, 8]. Pharmacotherapy in menstrual migraine is further complicated by the lack of strong evidencebased recommendation, the involvement of hormones, and in some cases the use of contraceptives [9].

Recent developments introduced several non-pharmacological neuromodulation approaches for the treatment of migraine [10]. The integration of neuromodulation into migraine care is recommended for suitable patients by both European and American Headache associations $[11,12]$. One of these is remote electrical neuromodulation (REN). REN stimulates mainly $\mathrm{C}$ and $\mathrm{A} \delta$ fibers in the upper arm to activate an endogenous analgesic mechanism termed conditioned pain modulation (CPM) [13-15] (see Fig. 1). Efficacy studies have demonstrated that REN is safe and clinically beneficial for the acute treatment of migraine in adults with episodic migraine [16-22], adults with chronic migraine [23], and adolescents with migraine [24]. A comparison of the efficacy of REN to that of acute migraine medications indicated that REN has non-inferior efficacy compared to the tested acute migraine therapies [20]. A recent meta-analysis 
a

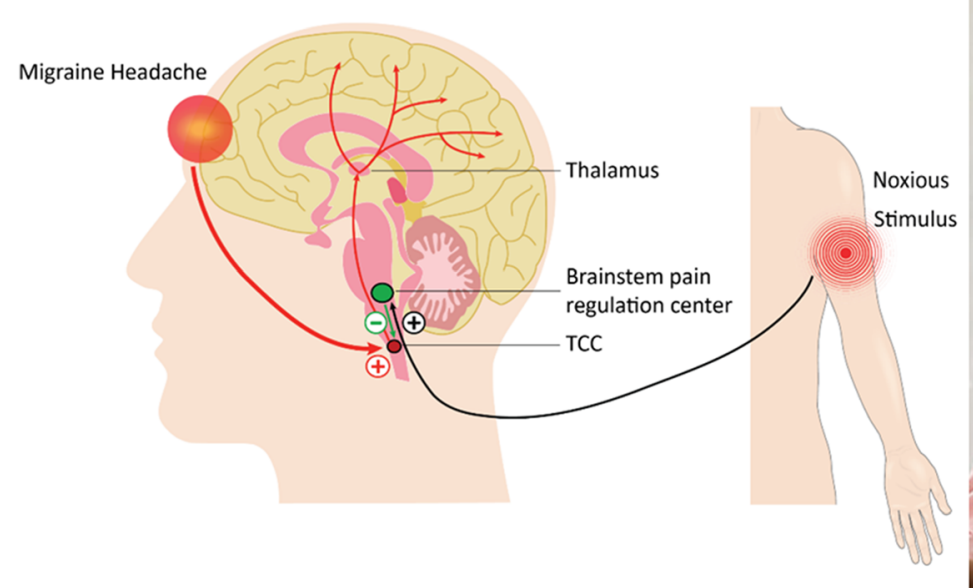

b

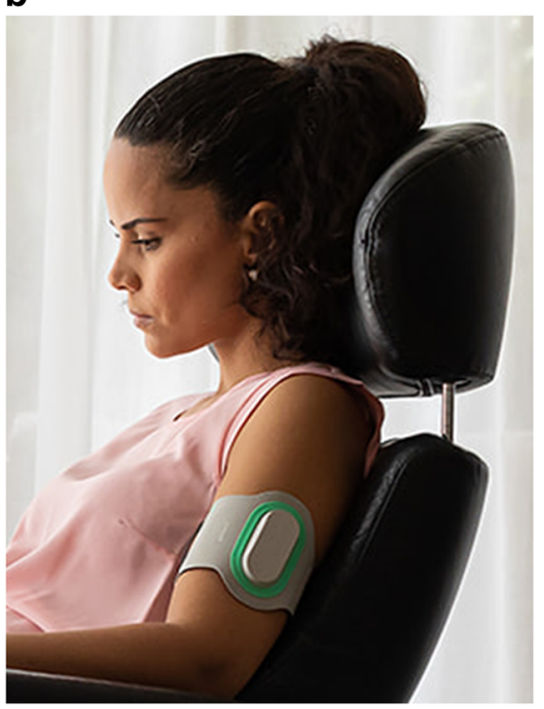

Fig. 1 REN's principle of operation. a Schematic illustration of the principle of operation of REN (modified with permission from Yarnitsky et al. [17]; Headache). The device stimulates $\mathrm{C}$ and $\mathrm{A} \delta$ noxious sensory fibers of the upper arm above their depolarization threshold yet below the perceived pain threshold. The noxious information reaches the brainstem through the ascending pain pathway (black). This information activates the descending pain inhibitory pathway (green), involving the brainstem pain

further found that REN was the only migraine neuromodulation intervention for which there is sufficient high-quality research, and thus the only one for which efficacy was well established [25].

The aim of this survey study was to evaluate the reported effectiveness, satisfaction, and safety of REN for the treatment of menstrual migraine in women who use REN as part of their regular migraine care. To investigate this, we conducted an online structured survey study among adult women who have been regularly using REN.

\section{METHODS}

\section{Study Design and Participants}

This was a retrospective, observational survey study in which users of the REN device (Nerivio $^{\circledR}$, Theranica Bio-Electronics Ltd., Netanya, regulation center (which includes the PAG, RVM, and $S R D$ ), which inhibit incoming messages of pain in the trigeminal cervical complex (TCC) that occur during a headache of a migraine attack (red). $P A G$ periaqueductal gray, $R V M$ rostral ventromedial medulla, $S R D$ subnucleus reticularis dorsalis, TCC trigeminal cervical complex. b An image of the device as it is worn on the arm during treatment

Israel) were contacted by an in-app notification and/or e-mail (provided by the users, during the sign-up process to the Nerivio app) and offered to participate. The study protocol (ClinicalTrial.Gov registration number NCT04600388) was reviewed and approved by the Western Institutional Review Board (WIRB) and was conducted according to Good Clinical Practice (GCP) and the Declaration of Helsinki guidelines.

The survey was sent to 455 female patients aged 18-55 across the United States who used Nerivio between October 1, 2019, and October 18,2020 , and completed at least four REN treatment sessions. The study sample represents a randomly selected sub-sample of Nerivio users who met the admission criteria. The sample size was calculated to achieve an approximate number of 100 completed surveys. Data were then excluded from the analysis if a patient's report indicated that she was not menstruating, 


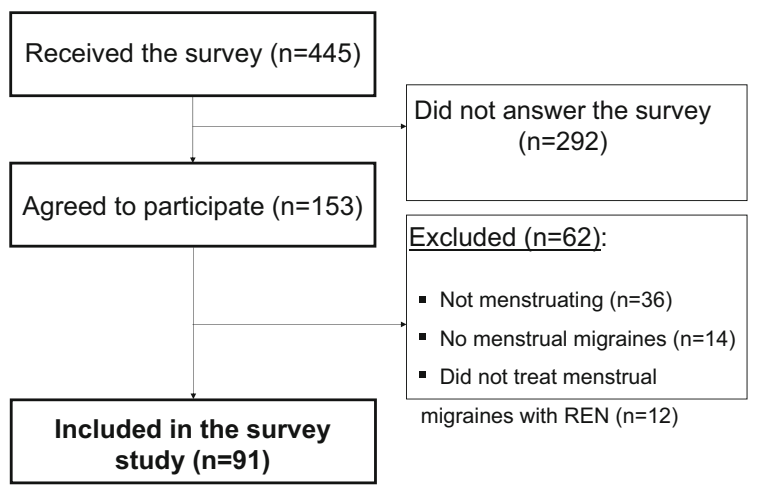

Fig. 2 Disposition chart

did not have menstrual migraines, or did not use the device to treat menstrual migraines (see Fig. 2 disposition chart). Menstrual migraine was defined according to the International Classification of Headache Disorders, third edition (ICHD-3; [2]), and reported as a yes/no question by the participants (Question text: "Do you usually experience migraines that occur 2 days before you get your period or during the first 3 days of your period (menstrual migraines)?"). Additionally, all participants were prescribed a migraine therapy by a neurologist.

Before taking the survey, patients provided informed consent. The consent form appeared as the first page of the survey, and participants clicked either "agree" or "disagree" to the consent statement (those who clicked "agree" proceeded to the survey, and those who clicked "disagree" were brought to an exit page). Patients received a $\$ 10$ gift card for participating in this survey. The first patient completed the survey on September 14, 2020, and the last patient completed the survey on October 20, 2020.

\section{The REN Device}

The REN device (Nerivio, Theranica Bio-Electronics Ltd., Netanya, Israel) and its mechanism of action have been described in detail elsewhere $[17,18]$. Briefly, this is a wearable device worn on the upper arm upon the onset of migraine symptoms, the device is controlled by a smartphone application (the Nerivio app). It stimulates mainly $\mathrm{C}$ and $\mathrm{A} \delta$ noxious fibers for $45 \mathrm{~min}$ to activate an endogenous analgesic mechanism termed conditioned pain modulation (CPM) [13-16]. The stimulus pulse frequency is set at $100-120 \mathrm{~Hz}$ (modulated), with a pulse width of $400 \mu \mathrm{s}$, and the output current is up to $40 \mathrm{~mA}$ that can be adjusted by the patient. The stimulus is a modulated, symmetrical, biphasic square pulse.

\section{Data Collection and Outcome Measures}

Participants were asked to complete a 5-min online survey assessing satisfaction, effectiveness, and safety-using multiple-choice questions, open-ended questions, and rating questions. The primary endpoints were: effectiveness of REN for menstrual migraine and user satisfaction. Secondary endpoints included reported tolerability of the treatment, side-effect prevalence, effectiveness for menstrual cramps, and effectiveness for pelvic pain.

\section{Data Analysis}

Continuous variables were analyzed using mean and standard deviation. Ordinal and nominal variables are presented as the total number and percentage of patients in each category, per question. The multiple-choice questions concerning effectiveness and tolerability (Q3, Q6, Q8, Q9) were rated from least to most, and for those questions we present results ranging from moderate to extreme together (i.e., answers 3-5), along with separate rates of each of the answers. Question 2 (satisfaction) was rated on a five-point Likert scale (i.e., the middle answer was neutral), and thus results are presented separately for negative answers $(1,2)$, neutral answer (3), and positive answers $(4,5)$, along with rates of each of the five answers.

Data were analyzed using Microsoft Office Excel version 16 (Microsoft Inc., USA), and IBM SPSS statistics software version 23.0. (SPSS Inc., USA). 


\section{RESULTS}

\section{Participants}

Out of 455 women who received the survey, 153 agreed to participate in the study. From those, the following were excluded (see Fig. 1 disposition chart): 36 participants who were not menstruating, 14 participants who did not have menstrual migraine, and 12 participants who did not use the device to treat menstrual migraines. This resulted in the inclusion of 91 participants in the analysis set. Participants were predominantly white $(92.3 \%$ [84/91]), had a mean age of $35.2 \pm 8.1$, had an average number of $13.3 \pm 8.7$ headache days per month, and treated $4.9 \pm 4.8$ menstrual migraines with Nerivio (all participants used Nerivio at least four times to treat migraines, regardless of menstruation). Based on patient reports (in accordance with ICHD-3 criteria [2]), $88(96.7 \%)$ women had menstrually related migraine (attacks occur especially but not only during the perimenstrual period [2]), and three women $(3.3 \%)$ had pure menstrual migraine (attacks occur only with menstruation [2]). This ratio is consistent with the relative ratio of women with pure menstrual migraine in previous epidemiological studies estimating the population of women with migraine, which is $4 \%$ [26].

\section{Menstrual Migraine Effectiveness}

\section{Effectiveness}

Of the participants, $74.7 \%(68 / 91)$ reported that the treatment was at least moderately effective (moderately effective $37.4 \%$, very effective $26.4 \%$, extremely effective $11.0 \%$ ). The treatment was reported not effective in $25.3 \%$ (23/ 91) of the participant (slightly effective: $20.9 \%$, not effective $4.4 \%$ ).

\section{Satisfaction}

Of the participants, $45.1 \%$ (41/91) reported satisfaction from REN (slightly satisfied 33\%, extremely satisfied $12.1 \%)$, while $34.1 \%(31 / 91)$ were neutral, and $20.9 \%(19 / 91)$ were not satisfied (slightly dissatisfied $16.5 \%$, not at all satisfied $4.4 \%)$.

\section{Cramps and Pelvic Pain}

\section{Menstrual Cramps}

Of the participants, $82.4 \%(75 / 91)$ indicated that they experience menstrual cramps. Out of these $75,38.7 \%(29 / 75)$ reported that REN was at least moderately effective (moderately effective $28.0 \%$, very effective $8 \%$, extremely effective $2.7 \%$ ) in treating the cramps. The treatment was reported not effective for cramps in $61.3 \%$ $(46 / 75)$ of the participant (slightly effective: $29.3 \%$, not effective $32.2 \%$ ).

\section{Pelvic Pain}

Of the 91 participants, 38 patients (41.8\%) indicated that they experience pelvic pain during menstruation. Out of these 38, 36.8\% (14/ 38 ) reported that REN was at least moderately effective for the pelvic pain (moderately effective $28.9 \%$, very effective $2.6 \%$, extremely effective $5.3 \%$ ). The treatment was reported not effective for pelvic pain by $63.2 \%(24 / 38)$ of the participant (slightly effective: $28.9 \%$, not effective $34.2 \%)$.

\section{Safety and Tolerability}

\section{Tolerability}

Of the participants, $100 \%$ (91/91) reported that the treatment was at least moderately tolerable (moderately tolerable $8.8 \%$, very tolerable $20.9 \%$, extremely tolerable $70.3 \%$ ).

\section{Side Effects}

Twelve patients (13.2\%) reported mild shortterm side effects: sore arm for several days after treatment $(n=1)$, bruise on the arm $(n=1)$, nausea $(n=2)$, dizziness $(n=1)$, drowsiness $(n=1)$, muscle twitching $(n=3)$, numbness in the arm during treatment $(n=2)$, and tingling in the arm and hand $(n=1)$.

\section{Use of Rescue Medications}

Sixty-nine of the participants reported their medication intake following REN treatments 
during the during the studied period. Of which, $40.9 \%(n=27)$ had never taken any medications within $2 \mathrm{~h}$ from the treatment onset in any of the REN treatments they have done. $66.7 \%(n=46)$ were medication free in at least $75 \%$ of the treatments, and $92.8 \%(n=64)$ were medication free in at least $50 \%$ of their REN treatments.

\section{DISCUSSION}

The current survey study suggests that REN may offer an effective, well-tolerated treatment option for menstrual migraine. The study assessed real-world experience with REN for acute treatment of menstrual migraines by capturing reported effectiveness, satisfaction, and tolerability rates of the intervention via an on-line structured survey.

Our results show that nearly $75 \%$ of the participants reported that the treatment was at least moderately effective (with $37.4 \%$ of participants reporting it either very effective, or extremely effective), suggesting that REN may provide a non-pharmacological treatment option for women with menstrual migraine. The current results join a line of clinical studies with similar results. In a double-blind shamcontrolled study in adults with episodic migraine [17], $66.7 \%$ of the participants achieved pain relief at $2 \mathrm{~h}$ post treatment (vs. $38.8 \%$ of participants in the sham group, with a therapeutic gain of $27.9 \% ; p<0.0001)$. Pain freedom and relief of most bothersome symptom were also significantly higher in the REN group compared to sham. A prospective, openlabel dual-center study in adults with chronic migraine [23] indicated that $73.7 \% \%$ of the participants achieved pain relief at $2 \mathrm{~h}$ post treatment. An open-label, single-arm, multicenter study in adolescents with migraine [24] also demonstrated pain relief in $71 \%$ of participants at $2 \mathrm{~h}$, and improved functional ability in $69 \%$ of the participants. It should be noted that in the current study, data was collected differently (i.e., via patients' retrospective global reports rather than after each treatment in a prospective clinical trial). However, while a randomized controlled trial is needed to further establish the therapeutic benefits, together these findings suggest that REN is an effective migraine treatment and could potentially offer much-needed relief in menstrual migraine as well.

Forty-five percent of the participants reported being either slightly satisfied or extremely satisfied (33.0\%, 12.1\%; respectively) more than twice the ratio of unsatisfied participants which was $20.9 \%$ (slightly dissatisfied $16.5 \%$, not at all satisfied $4.4 \%)$. We acknowledge that while satisfaction rates were substantial, they are lower than effectiveness rates. This could reflect the fact that satisfaction is a complex construct, potentially combining factors like expectations for clinical efficacy, coverage by insurance, cost considerations, etc. We have chosen to assess both effectiveness and satisfaction, since they are both recommended endpoints according to the International Headache Society [27]. The finding that a considerable portion of the participants consider the treatment effective and satisfactory provides important information, as menstrual migraine attacks are typically more debilitating and less responsive to current acute treatments $[4,28]$.

All participants (100\%) reported that the intervention was at least moderately tolerable (with 70.3\% reporting it extremely tolerable, and $20.9 \%$ reporting it very tolerable). Twelve participants (13.2\%) reported mild short-term side effects, most of which were expected and have been observed in previous studies $[17,23]$. These results provide further support for the favorable tolerability and safety profile of REN found in clinical trials in episodic and chronic migraine in adults [16-21, 23], and adolescents [24].

Lastly, the study also examined whether REN treatment was reported to have an effect on two additional pain syndromes that afflict women during menstruation: menstrual cramps [29] and pelvic pain [30]. Nearly $40 \%$ of participants reported at least moderate effectivity for both symptoms (cramps $38.7 \%$, pelvic pain $36.8 \%$ ). while REN was not as highly effective for those symptoms as reported for migraine, the results suggest that it may offer a moderate degree of relief from cramps and pelvic pain for some patients. It should be noted that patients were 
not instructed to treat these syndromes, and effectivity might possibly improve if treated at onset (similarly to treatment instructions for migraine).

It should be noted that the effectivity and tolerability questions ranged from null to max (i.e., from 'not effective' to 'extremely effective'), and thus the middle point of these scales was 'moderately effective' rather than 'neutral' that is often used in Likert scales. While a differently phrased scale could have resulted in a different breakdown of the results, we believe that this scale best suits the effectivity range, and we present it along with full specification of each category in the scale (e.g., moderately effective $37.4 \%$, very effective $26.4 \%$, extremely effective $11.0 \% \ldots$ ) for each question. Several limitations of this study should be noted. First, the retrospective self-report nature of the study may limit the conclusions of this survey by recall bias. While this is true, this study provides a naturalistic perspective on the effects of the treatment, and reflects the real-life perceptions that patients have on the intervention, in their own natural environment. The study is thus not intended to replace a randomized clinical trial (and is not presented as one), but rather to present the participants' reports on their regular migraine care. Second, the diagnosis of menstrual migraine relied on self-report rather than a physician diagnosis. However, the report questions were identical to the ICHD-3 diagnosis criteria [2], and were answered as a yes/no response similar to the way a physician would diagnose. Lastly, only individuals who completed at least four REN treatments were sent a survey (possibly creating a selection bias). The sample thus represents REN users and is not meant to reflect the general population.

\section{CONCLUSIONS}

Menstrual migraine is more severe and harder to treat than non-menstrual migraine. The current survey study indicates that REN may provide a safe, non-pharmacological treatment option for women with menstrual migraine. While randomized controlled trials are needed to further establish the therapeutic benefits of REN in menstrual migraine, this study indicates that patients find REN effective and very well tolerated. It thus provides for the first-time evidence supporting this non-pharmacological treatment for menstrual migraine, for a population that long-awaits medical solutions.

\section{ACKNOWLEDGEMENTS}

We thank the subjects for their participation in the study.

Funding. This study and the journal's Rapid Service Fee were funded by Theranica Bio-Electronics Ltd.

Authorship. All named authors meet the International Committee of Medical Journal Editors (ICMJE) criteria for authorship for this article, take responsibility for the integrity of the work as a whole, and have given their approval for this version to be published.

Authorship Contributions. All authors participated in the interpretation of the data and revising of the manuscript, and contributed important intellectual content. All authors have approved the final version of the manuscript. Dr. Rabany and Dr. Nierenburg drafted the manuscript. Dr. Rabany, Dr. Nierenburg, and Mr. Ironi designed the analysis. Dr. Rabany performed the analysis. Dr. Lin, Mr. Ironi and Dr. Harris created the study concept and wrote the survey. Dr. Lin, Mr. Ironi, and Dr. Harris participated in the acquisition of the data.

Medical Writing, Editorial, and Other Assistance. Ms. Shira Tamir - an employee of Theranica Bio-Electronics, contributed to this article in specific aspects of data preparation and processing, as well as assistance in the submission process.

Disclosures. Linus Chuang declares that he has no conflict of interest.

Hida Nierenberg has received honoraria from Allergan and from Theranica Bio-Electronics.

Hida Nierenberg and Paul Wright are employees of the Nuvance Health Neurosciences Insti- 
tute which had received a $\$ 15,000$ educational grant for neuroscience medical education from Theranica Bio-Electronics, to educate clinicians about applying neuromodulation devices to treat neurological conditions and diseases.

Roni Sharon has been a paid consultant of Theranica Bio-Electronics.

Liron Rabany, Tamar Lin, Dagan Harris, and Alon Ironi are employees of Theranica BioElectronics.

Compliance with Ethics Guidelines. The study protocol (ClinicalTrial.Gov registration number NCT04600388) was reviewed and approved by Western Institutional Review Board (WIRB) and was conducted according to Good Clinical Practice (GCP) and the Declaration of Helsinki guidelines. Before taking the survey, patients provided informed consent.

Data Availability. The datasets generated during and/or analyzed during the current study are available from the corresponding author on reasonable request.

Open Access. This article is licensed under a Creative Commons Attribution-NonCommercial 4.0 International License, which permits any non-commercial use, sharing, adaptation, distribution and reproduction in any medium or format, as long as you give appropriate credit to the original author(s) and the source, provide a link to the Creative Commons licence, and indicate if changes were made. The images or other third party material in this article are included in the article's Creative Commons licence, unless indicated otherwise in a credit line to the material. If material is not included in the article's Creative Commons licence and your intended use is not permitted by statutory regulation or exceeds the permitted use, you will need to obtain permission directly from the copyright holder. To view a copy of this licence, visit http://creativecommons.org/licenses/by$\mathrm{nc} / 4.0 /$.

\section{REFERENCES}

1. Steiner TJ, Stovner LJ, Jensen R, Uluduz D, Katsarava Z, on behalf of Lifting The Burden: the Global Campaign against Headache. Migraine remains second among the world's causes of disability, and first among young women: findings from GBD2019. J Headache Pain. 2020;21(1):137.

2. Headache Classification Committee of the International Headache Society. The international classification of headache disorder, 3rd edition. Cephalalgia. 2018;38(1):1-211.

3. Vetvik KG, MacGregor EA, Lundqvist C, Russell MB. Self-reported menstrual migraine in the general population. J Headache Pain. 2010;11(2):87-92.

4. Maasumi K, Tepper SJ, Kriegler JS. Menstrual migraine and treatment options: review. Headache J Head Face Pain. 2017;57(2):194-208.

5. Ansari T, Lagman-Bartolome AM, Monsour D, Lay C. Management of menstrual migraine. Curr Neurol Neurosci Rep. 2020;20(10):1-6.

6. Pinkerman B, Holroyd K. Menstrual and nonmenstrual migraines differ in women with menstruallyrelated migraine. Cephalalgia. 2010;30(10): 1187-94.

7. Diener H-C, Holle D, Solbach K, Gaul C. Medication-overuse headache: risk factors, pathophysiology and management. Nat Rev Neurol. 2016;12(10):575-83.

8. Vandenbussche N, Laterza D, Lisicki M, Lloyd J, Lupi C, Tischler $\mathrm{H}$, et al. Medication-overuse headache: a widely recognized entity amidst ongoing debate. J Headache Pain. 2018;19(1):50.

9. Martelletti P, Guglielmetti M. Approaching the appropriate pharmacotherapy of menstrual migraine. Expert Rev Neurother. 2020;20(1):1-2.

10. Hou AY, Chen AY, Yuan H, Silberstein SD. Peripheral neuromodulation for the treatment of migraine and headache: recent advances. Bioelectron Med. 2019;2(4):151-62.

11. The American Headache Society. The American Headache Society position statement on integrating new migraine treatments into clinical practice. Headache J Head Face Pain. 2019;59(1):1-18.

12. Martelletti P, Jensen RH, Antal A, Arcioni R, Brighina $\mathrm{F}$, de Tommaso $\mathrm{M}$, et al. Neuromodulation of chronic headaches: position statement from the European Headache Federation. J Headache Pain. 2013;14(1):86. 
13. Nir RR, Yarnitsky D. Conditioned pain modulation. Curr Opin Support Palliat Care. 2015;9(2):131-7.

14. Nirl R-R, Granovskyl Y, Yarnitskyl D, Sprecherl E, Granotl M. A psychophysical study of endogenous analgesia: the role of the conditioning pain in the induction and magnitude of conditioned pain modulation. Eur J Pain. 2011;15(5):491-7.

15. Yarnitsky D, Granot $M$, Nahman-Averbuch $H$, Khamaisi M, Granovsky Y. Conditioned pain modulation predicts duloxetine efficacy in painful diabetic neuropathy. Pain. 2012;153(6):1193-8.

16. Yarnitsky D, Volokh L, Ironi A, Weller B, Shor M, Shifrin A, et al. Nonpainful remote electrical stimulation alleviates episodic migraine pain. Neurology. 2017;88(13):1250-5.

17. Yarnitsky D, Dodick DW, Grosberg BM, Burstein R, Ironi A, Harris D, et al. Remote electrical neuromodulation (REN) relieves acute migraine: a randomized, double-blind, placebo-controlled, multicenter trial. Headache J Head Face Pain. 2019;59(8):1240-52.

18. Rapoport AM, Lin T. Device profile of the Nerivio ${ }^{\mathrm{TM}}$ for acute migraine treatment: overview of its efficacy and safety. Expert Rev Med Devices. 2019;16(12):1017-23.

19. Marmura MJ, Lin T, Harris D, Ironi A, Rosen NL. Incorporating Remote Electrical Neuromodulation (REN) Into Usual Care Reduces Acute Migraine Medication Use: An Open-Label Extension Study. Front Neurol [Internet]. 2020. Available from: https://www.frontiersin.org/articles/https://doi.org/ 10.3389/fneur.2020.00226/full?\&utm_source=Email_ to_authors_\&utm_medium=Email\&utm_content= T1_11.5e1_author\&utm_campaign=Email_publica tion\&field=\&journalName=Frontiers_in_Neurology \&id=496800 Accessed 2020 Apr 7

20. Rapoport AM, Bonner JH, Lin T, Harris D, Gruper Y, Ironi A, et al. Remote electrical neuromodulation (REN) in the acute treatment of migraine: a comparison with usual care and acute migraine medications. J Headache Pain. 2019;20(1):83.

21. Tepper SJ, Lin T, Montal T, Ironi A, Dougherty C. Real-world Experience with Remote Electrical Neuromodulation in the Acute Treatment of Migraine. Pain Med [Internet]. Available from: https://academic.oup.com/painmedicine/advance-article/doi/ https://doi.org/10.1093/pm/pnaa299/5906300

Accessed 2020 Sep 17

22. Hershey AD, Lin T, Gruper Y, Harris D, Ironi A, Berk $\mathrm{T}$, et al. Remote electrical neuromodulation for acute treatment of migraine in adolescents. Headache J Head Face Pain. 2021;61(2):310-7.

23. Nierenburg H, Vieira JR, Lev N, Lin T, Harris D, Vizel M, et al. Remote electrical neuromodulation for the acute treatment of migraine in patients with chronic migraine: an open-label pilot study. Pain Ther. 2020;9:531.

24. Hershey AD, Lin T, Gruper Y, Harris D, Ironi A, Berk $\mathrm{T}$, et al. Remote electrical neuromodulation for acute treatment of migraine in adolescents. Headache J Head Face Pain. 2020;61:310.

25. Moisset X, Pereira B, Ciampi de Andrade D, Fontaine D, Lantéri-Minet M, Mawet J. Neuromodulation techniques for acute and preventive migraine treatment: a systematic review and meta-analysis of randomized controlled trials. J Headache Pain. 2020;21(1):142.

26. Vetvik KG, MacGregor EA, Lundqvist C, Russell MB. Prevalence of menstrual migraine: a populationbased study. Cephalalgia. 2014;34(4):280-8.

27. Tassorelli C, Diener H-C, Dodick DW, Silberstein SD, Lipton RB, Ashina M, et al. Guidelines of the International Headache Society for controlled trials of preventive treatment of chronic migraine in adults. Cephalalgia. 2018;38(5):815-32.

28. MacGregor EA, Victor TW, Hu X, Xiang Q, Puenpatom RA, Chen W, et al. Characteristics of menstrual vs nonmenstrual migraine: a post hoc, within-woman analysis of the usual-care phase of a nonrandomized menstrual migraine clinical trial. Headache J Head Face Pain. 2010;50(4):528-38.

29. Grandi G, Ferrari S, Xholli A, Cannoletta M, Palma F, Romani C, et al. Prevalence of menstrual pain in young women: what is dysmenorrhea? J Pain Res. 2012;20(5):169-74.

30. Mathias SD, Kuppermann M, Liberman RF, Lipschutz RC, Steege JF. Chronic pelvic pain: prevalence, health-related quality of life, and economic correlates. Obstet Gynecol. 1996;3(87):321-7. 